\title{
Interhemispheric Stroop-Like Interference in Number Comparison: Evidence for Strong Interhemispheric Integration of Semantic Number Information
}

\author{
Elie Ratinckx \\ Ghent University
}

\author{
Marc Brysbaert \\ Ghent University and University of London
}

\begin{abstract}
Three experiments investigated interhemispheric interactions in number comparison using the interhemispheric Stroop-like paradigm (E. Ratinckx, M. Brysbaert, \& B. Reynvoet, 2001). In all experiments, a target was presented in 1 visual field simultaneously with a distractor in the other visual field. In Experiment 1, both target and distractor were of the same modality (Arabic digits), whereas in Experiment 2, target and distractor were of different modalities (Arabic digits and word numerals). In Experiment 3, the interhemispheric Stroop-like task of Experiment 1 was combined with intrahemispheric conditions to evaluate the strength of the interhemispheric interactions. Overall, the results point to strong interhemispheric integration during semantic access and response preparation with very weak lateralization of the semantic number system.
\end{abstract}

Comparing two numbers is the most basic semantic task in numerical cognition. In most studies, a participant simply categorizes a visually presented number (e.g., an Arabic digit) as larger or smaller than a specified reference number (see, e.g., Dehaene, Dupoux, \& Mehler, 1990). Although the physical resemblance of the Arabic digits 5 and 6 is not larger than that of 5 and 9, a numerical distance effect emerges. Moyer and Landauer (1967) were the first to show this effect: Participants took more time to select the larger numerical value of two simultaneously presented digits when the numerical distance was small (e.g., between 5 and 6) than when it was large (e.g., between 5 and 9). Similar semantic effects have been reported for young children (e.g., Sekuler \& Mierkiewicz, 1977) and for a variety of animal species (for an overview, see Dehaene, DehaeneLambertz, \& Cohen, 1998), suggesting that humans share a fundamental property of magnitude estimation with other species. Therefore, the numerical distance effect is often taken as a basic characteristic of elementary numerical cognition (Schwarz \& Ischebeck, 2000).

Studies with split-brain patients (e.g., Cohen \& Dehaene, 1996; Seymour, Reuter-Lorenz, \& Gazzaniga, 1994) as well as brain-imaging studies (e.g., Chochon, Cohen, van de

Elie Ratinckx, Department of Experimental Psychology, Ghent University, Ghent, Belgium; Marc Brysbaert, Department of Experimental Psychology, Ghent University, and Department of Psychology, Royal Holloway, University of London, London, England.

We thank Antoine Tavernier for his technical support and Nathalie De Ridder for running Experiment 2. We also thank Wim Fias and Voyko Kavcic for helpful comments on an earlier version of this article.

Correspondence concerning this article should be addressed to Elie Ratinckx, Department of Experimental Psychology, Ghent University, Henri Dunantlaan 2, B-9000 Ghent, Belgium. E-mail: elie.ratinckx@rug.ac.be
Moortele, \& Dehaene, 1999; Pesenti, Thioux, Seron, \& De Volder, 2000) have indicated that number magnitude is bilaterally represented in the brain. More specifically, most brain-imaging studies suggest the involvement of the inferior parietal region of both the left hemisphere (LH) and the right hemisphere (RH; e.g., Pinel et al., 1999) in accessing the semantic code needed for number comparison and other semantic manipulations of numerical quantities.

Behavioral research with normal participants has also pointed to the existence of rich interhemispheric interactions in number comparison together with the existence of weak hemispheric asymmetries. In an interhemispheric Stroop-like task, Ratinckx et al. (2001) presented a target in one visual half field (VHF) simultaneously with a distractor of the same format in the other VHF. Participants had to indicate manually whether the magnitude of the target digit was large (5 or 6 ) or small ( 1 or 2$)$, ignoring the distractor stimulus $(1,2,5$, or 6$)$. Significant bilateral field interactions of the target and the distractor were found both at an early level of processing and at a later response level. Reaction times (RTs) were faster when target and distractor had the same value than when they had a different value but were associated with the same response (an identity effect). Trials in which target and distractor were associated with the same response resulted in faster RTs than trials in which target and distractor were associated with different responses (demonstrating a response compatibility effect). Other evidence for the importance of interhemispheric integration in number comparison has been reported by Banich and Belger (1990). They showed that performance in a number comparison task was better when the two digits were presented in different VHFs than when they were presented in the same VHF. In contrast, when the task involved a judgment about the physical identity of the Arabic numerals, unilateral presentation yielded faster responses than bilateral presentation. 
Other than the presence of strong interhemispheric interactions in number comparison, only weak evidence has been found for hemispheric lateralization. After a review of the literature based on behavioral VHF studies, Ratinckx et al. (2001) concluded that number comparison yields considerably less laterality effects than other numerical tasks such as odd-even judgment or number recognition (see also Boles, 1986). This finding was in line with Ratinckx et al.'s own subsequent data and with one split-brain study in which the same semantic distance effect was obtained for both hemispheres (Cohen \& Dehaene, 1996). The data were also in line with the mixed evidence that comes from research with patients (e.g., Cohen \& Dehaene, 1996; Dahmen, Hartje, Buessing, \& Sturm, 1982; Rosselli \& Ardila, 1989) and brain-imaging studies in which sometimes a left (e.g., Pesenti et al., 2000; Pinel et al., 1999) and sometimes a right laterality pattern (e.g., Chochon et al., 1999; Dehaene, 1996) is reported.

Together, these findings are in line with Dehaene's (1992) neuroanatomical model of number processing, which assumes that the analogical number-magnitude representation has a copy in the $\mathrm{LH}$ and $\mathrm{RH}$ and that both copies are linked via transcallosal pathways.

\section{The Present Study}

As reported above, Ratinckx et al. (2001) observed interhemispheric interactions at an early stage of processing and at a later response level in a number comparison task. However, their design did not allow them to determine whether the early interhemispheric interactions were perceptual or semantic, because only conditions in which target and distractor were identical (i.e., 1-1, 2-2, 5-5, and 6-6) were compared with conditions in which target and distractor differed by one (i.e., 1-2, 2-1, 5-6, and 6-5).

To test whether interhemispheric integration in number comparison is situated at the semantic level or at the perceptual level, we designed two new manual RT experiments (Experiments 1 and 2). In both, the participants' task was to evaluate whether a target number was smaller (1,2, and 3$)$ or larger $(5,6$, and 7$)$ than 4 . We used a variant of Lambert's (1993) interhemispheric Stroop-like task. The target stimulus was presented randomly in the right visual field (RVF) or in the left visual field (LVF), together with a distractor in the opposite VHF. Participants were asked to react to the target number and to ignore the distractor. This technique with bilateral stimulus display not only allowed us to look for interhemispheric interactions but is also recommended by several authors as the best way to obtain behavioral evidence of cerebral asymmetries (see, e.g., Boles, 1986, 1987, 1990; Hellige \& Sergent, 1986). In Experiment 1, target and distractor were of the same modality (Arabic digits), whereas in Experiment 2, target and distractor were of different modalities (Arabic digits vs. word numerals).

The design of the experiments allowed us to distinguish between interhemispheric interactions at three different levels: response, semantic, and perceptual. Interactions at the response level are revealed by the response compatibility effect (see also Dehaene, Naccache, et al., 1998; Koechlin,
Naccache, Block, \& Dehaene, 1999). To assess this effect, we compared trials in which the target and distractor elicited the same response (congruent trials) with trials in which target and distractor required different responses (incongruent trials). In Experiment 1, where target and distractor belonged to the same modality, we evaluated semantic priming by looking at a decrease in RTs as a function of the numerical distance between the target and the distractor in the congruent trials. Several studies (e.g., Brysbaert, 1995; Koechlin et al., 1999) have shown that RTs to numerals are faster when, immediately before, a prime with a close value (distance of 1) is shown than when a prime with a more distant value (e.g., distance of 2) is shown. This effect suggests that the prime has activated a semantic representation and that activation has spread to the nearby representations. The effect of perceptual priming was determined by investigating whether the difference between distance of 0 and distance of 1 was larger than the difference between distance of 1 and distance of 2 . At a distance of 0 , target and distractor are identical, so that in this condition there is a possibility of perceptual priming next to semantic priming. Evidence for perceptual priming was reported by Reynvoet and Brysbaert (1999). They asked participants to name target digits, that were preceded by masked primes. When prime and target were identical (i.e., distance of 0 ), target naming time was $444 \mathrm{~ms}$; when prime and target differed by 1 , naming time was $478 \mathrm{~ms}$; and when prime and target differed by 2 , naming time was $487 \mathrm{~ms}$. Thus, the difference between distances of 0 and 1 (34 ms) was significantly larger than the difference between distances of 1 and 2 ( 9 $\mathrm{ms}$ ), indicating the presence of perceptual priming in addition to semantic priming. In Experiment 2 of the present study, we further investigated semantic priming by comparing numbers of different modalities. When target and distractor belong to a different modality, semantic priming cannot be due to perceptual overlap.

In Experiment 3 we compared the interhemispheric Stroop-like conditions of Experiment 1 with intrahemispheric conditions in which target and distractor were presented in the same VHF. Such a design allowed us to compare the interhemispheric connections with intrahemispheric connections. In addition, the unilateral conditions gave us a further possibility to find differences in number processing between the left and the right cerebral hemisphere by comparing the semantic priming effects in LVF and RVF. Finally, Experiment 3 also included a neutral distractor, allowing us to investigate to what extent the effects in our Stroop-like task were due to interference or facilitation.

\section{Experiment 1}

To further investigate interhemispheric integration in number comparison, we ran a first experiment in which target and distractor were of the same modality (Arabic numerals). The stimulus set was large enough that we could disentangle the effect of perceptual priming from the effect of semantic priming (see above). 


\section{Method}

Participants. Twelve undergraduates from Ghent University participated for course credit. All participants (age range $=18-34$ years) were men and were right-handed as confirmed by a Dutch translation of the Oldfield (1971) questionnaire. Participants were unaware of the purpose of the experiment and had normal or corrected-to-normal vision.

Procedure. Stimuli were presented on a Pentium PC connected to a 15-in. color Yakumo monitor. Responses were measured with an external four-key board connected to the gameport of the PC. Stimulus presentation and response timing were measured to the nearest millisecond with the software routines of Brysbaert and colleagues (Bovens \& Brysbaert, 1990; Brysbaert, 1990; Brysbaert, Bovens, d'Ydewalle, \& Van Calster, 1989).

The fixation stimulus consisted of two short vertical lines of $0.4^{\circ}$, one above the other, separated by a gap of $0.6^{\circ}$. A stimulus display consisted of a target stimulus presented in one VHF, an arrow presented in the gap of the fixation mark pointing to the target, and a distractor stimulus presented in the opposite VHF. A trial started with a fore period of $1,000 \mathrm{~ms}$, followed by the presentation of the fixation stimulus, a second fore period of 500 $\mathrm{ms}$, and the stimulus display shown for $100 \mathrm{~ms}$.

Stimuli were the Arabic numerals 1, 2, 3, 5, 6, and 7, extending $0.3^{\circ} \times 0.4^{\circ}$ horizontally and vertically. Both targets and distractors were presented $1.4^{\circ}$ left or right of the fixation mark (measured from the center of the stimulus to the vertical line of the fixation mark).

The participants were instructed to fixate the gap of the fixation mark and to judge, as quickly and accurately as possible, whether the target numeral was smaller or larger than 4 . The participants sat at a distance of about $60 \mathrm{~cm}$ from the screen (there were no head restraints).

Participants' responses were bimanual to avoid stimulus-response compatibility effects. The response box consisted of four buttons, arranged in such a way that they could easily be pressed with digits and middle fingers (i.e., the two outer buttons were placed $15 \mathrm{~mm}$ above the inner buttons). Half of the participants were instructed to press the two upper buttons simultaneously with the middle fingers of the right and the left hand when the target was larger than 4 and to press the lower buttons with the digits when the target was smaller than 4 . The other half of the participants were given the opposite stimulus-response assignment. Participants were instructed to ignore the distractor stimulus.

Each participant completed a training block of 72 trials and four experimental blocks of 288 trials. This resulted in a total of 16 observations per participant in each of the $2 \times 6 \times 6(\mathrm{VHF} \times$ Target Value $\times$ Distractor Value $)=72$ repeated measures conditions. At the end of each block the participants received feedback about their RTs and accuracy.

\section{Results}

Only average RTs of correct responses in the range of $150-1,500 \mathrm{~ms}$ were analyzed. Error rate did not exceed $15 \%$ per participant $(M=9 \%)$. Additionally, the percentage of outliers, averaged across participants, amounted to only $0.1 \%$ of the correct responses. There was no speed-accuracy trade-off, as indicated by a positive correlation between $\mathrm{RT}$ and number of errors over the 72 cells of the design $(r=$ $.69, n=72, p<.05)$.

A $2 \times 6 \times 6$ analysis of variance (ANOVA) was performed with the following repeated measures: VHF (2 levels) and target and distractor values (6 levels each).

The main effect of VHF was not significant $(\mathrm{LVF}=588$ $\mathrm{ms}, \mathrm{RVF}=583 \mathrm{~ms} ; F<1)$. There was a significant main effect of target value $(1=569 \mathrm{~ms}, 2=581 \mathrm{~ms}, 3=608 \mathrm{~ms}$, $5=602 \mathrm{~ms}, 6=584 \mathrm{~ms}, 7=569 \mathrm{~ms}), F(5,55)=14.02$, $M S E=2,819.57, p<.01$, largely due to the numerical distance effect (see Moyer \& Landauer, 1967): The RTs to the extreme values ( 1 and 7) were significantly faster than the RTs to the values 3 and 5, as revealed by post hoc comparisons (Tukey $p<.01$ ).

The main effect of distractor value was also significant $(1=588 \mathrm{~ms}, 2=579 \mathrm{~ms}, 3=583 \mathrm{~ms}, 5=585 \mathrm{~ms}, 6=$ $581 \mathrm{~ms}, 7=597 \mathrm{~ms}), F(5,55)=2.80, M S E=2,086.54$, $p<.03$. Post hoc comparisons showed that RTs with the distractor value 2 were significantly faster than with value 7 (Tukey $p<.02$ ).

Significant interactions between target and distractor value were observed, $F(25,275)=7.56, M S E=1,111.28$, $p<.01$, indicating interhemispheric interactions (see Table 1 for mean RTs). The origin of this interaction is examined below (see distance priming and response compatibility effect).

There was also a significant interaction between target and VHF, $F(5,55)=3.16, M S E=1,366.15, p<.02$ (see Figure 1). For small digits (1, 2, and 3) a slight LVF advantage was present $(\mathrm{LVF}=583 \mathrm{~ms}$ vs. $\mathrm{RVF}=588 \mathrm{~ms})$, whereas for larger digits $(5,6$, and 7) an RVF advantage seemed to emerge $(\mathrm{LVF}=593 \mathrm{~ms}$ vs. $\mathrm{RVF}=578 \mathrm{~ms})$. However, no VHF advantage was significant (Tukey $p>$ $.05)$.

To test response compatibility, we performed a $2 \times 2$ ANOVA with VHF ( 2 levels) and response compatibility (2 levels) as repeated measures. The main effect of VHF was

Table 1

Mean Reaction Times in Milliseconds as a Function of Target and Distractor (Experiment 1: Arabic Targets and Arabic Distractors)

\begin{tabular}{clccccc}
\hline & \multicolumn{7}{c}{ Distractor } \\
\cline { 2 - 6 } Target & \multicolumn{1}{c}{1} & \multicolumn{1}{c}{3} & \multicolumn{1}{c}{5} & \multicolumn{1}{c}{6} & 7 \\
\hline 1 & $538(5)$ & $548(4)$ & $567(3)$ & $583(7)$ & $577(8)$ & $598(11)$ \\
2 & $586(7)$ & $543(4)$ & $569(5)$ & $596(9)$ & $587(9)$ & $603(11)$ \\
3 & $609(17)$ & $593(14)$ & $594(9)$ & $618(10)$ & $618(12)$ & $618(12)$ \\
5 & $619(14)$ & $610(12)$ & $595(11)$ & $576(9)$ & $602(12)$ & $613(16)$ \\
6 & $600(14)$ & $595(13)$ & $591(10)$ & $569(7)$ & $550(6)$ & $598(8)$ \\
7 & $578(13)$ & $584(10)$ & $583(13)$ & $568(5)$ & $553(5)$ & $550(3)$ \\
\hline
\end{tabular}

Note. Values in parentheses are percentages of errors. 


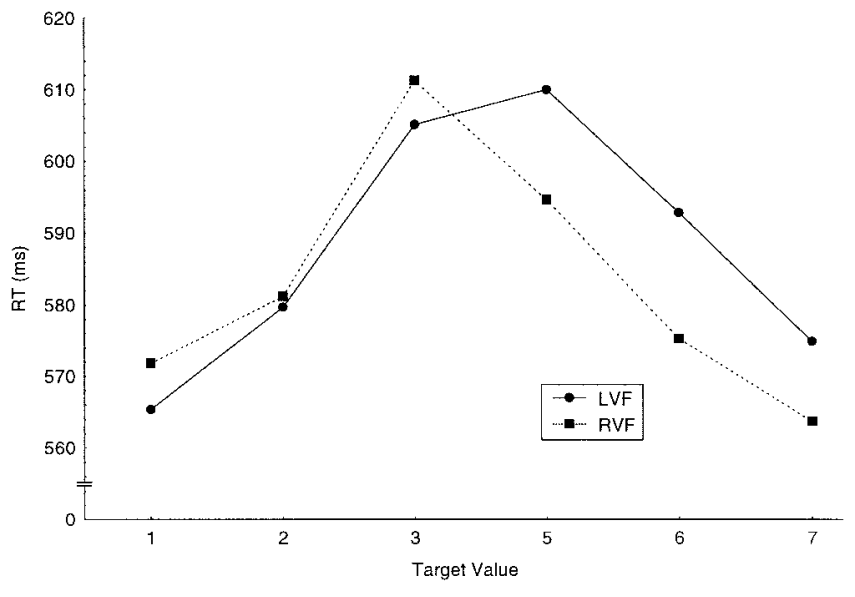

Figure 1. Significant interaction between visual half field and target value in Experiment 1 with Arabic numerals. $\mathrm{RT}=$ reaction time; $L V F=$ left visual field; $R V F=$ right visual field.

not significant $(F<1)$, which repeats the zero effect in the previous ANOVA. Response compatibility was significant, $F(1,11)=20.21, M S E=358.08, p<.01$, showing faster RTs in the congruent condition (572 ms) than in the incongruent condition $(597 \mathrm{~ms}$, see Table 2 for an overview of the mean effects). The interaction between VHF and response compatibility was not significant $(F<1)$.

To test the effects of semantic and perceptual priming, we ran a $2 \times 3$ ANOVA with VHF (2 levels) and distance between target and distractor ( 3 levels: distance of 0,1 , and 2 ) as repeated measures. Only observations for the targets 1 , 3 , 5, and 7 were included in the analysis to obtain an equivalent distribution of targets in all distance conditions. The main effect of VHF was not significant $(F<1)$, but the main effect of distance was significant, $F(2,22)=15.34$, $M S E=230.81, p<.01$, showing slower RTs with increasing distance between target and distractor (distance of $0=$ $563 \mathrm{~ms}$; distance of $1=572 \mathrm{~ms}$; distance of $2=587 \mathrm{~ms}$; see Table 2 for an overview of the effects). Planned comparisons returned a marginally significant difference between distance of 0 and distance of $1(p=.09)$ and a significant difference between distance of 1 and distance of $2(p<.01)$, revealing semantic priming. To investigate the presence of perceptual priming, we tested whether there was evidence for a quadratic component in addition to a linear component in the distance effect. The linear component represents semantic priming, whereas a significant quadratic component would indicate that the difference between distance of 0 and distance of 1 is larger than the difference between distance of 1 and distance of 2, pointing to the presence of perceptual priming. The linear trend was significant, $F(1,11)=24.31, M S E=285.67, p<.05$, whereas the quadratic trend failed to reach significance $(F<1)$. Finally, the interaction between VHF and distance was marginally significant, $F(2,22)=3.21, M S E=197.14$, $p=.06$. There was no difference between distance of 0 and distance of 1 when the target was displayed in the LVF (distance of $0=569 \mathrm{~ms}$; distance of $1=569 \mathrm{~ms}$; distance of $2=592 \mathrm{~ms}$ ), whereas in RVF the RTs seemed to differ for all distances (distance of $0=557 \mathrm{~ms}$; distance of $1=$ $576 \mathrm{~ms}$; distance of $2=583 \mathrm{~ms}$ ).

\section{Discussion}

As expected from previous research (Ratinckx et al., 2001), significant bilateral field interactions between the target and the distractor were obtained, showing rich interhemispheric interactions in number comparison. First, there was a response compatibility effect: Responses were on average $25 \mathrm{~ms}$ faster when target and distractor elicited the same response (small-small or large-large) than when they asked for different responses (small-large or large-small). Second, in the congruent trials, response times were $9 \mathrm{~ms}$ faster when target and distractor had the same value (e.g., 1-1) than when they differed by 1 unit (e.g., 1-2). The latter condition in turn was responded to $15 \mathrm{~ms}$ faster than the condition in which target and distractor differed by 2 units (e.g., 1-3). The absence of a quadratic trend and the significance of a linear trend in the distance effect strongly suggest that the interactions were situated at the semantic level and not at the perceptual level. In line with Ratinckx et al. (2001), there was no clear VHF asymmetry, suggesting an equivalent bilateral representation of numerical magnitude information. The only effect of VHF that approached significance was a tendency toward an LVF advantage for small numbers and an RVF advantage for large numbers. A similar finding was reported by Brysbaert (1995, Experiment 3 ), who noticed that participants reacted faster on trials in which numbers were presented in an ascending order (small numbers to the left and large numbers to the right) than in trials with numbers presented in a descending order. $\mathrm{He}$ attributed this finding to the hypothetical left-right orientation of the semantic number line (Dehaene, Bossini, \& Giraux, 1993; Fias, Brysbaert, Geypens, \& d'Ydewalle, 1996), so that an ascending presentation of digits is in

Table 2

Distance Effects and Response Compatibility Effects in Experiments 1, 2, and 3

\begin{tabular}{lccc}
\hline $\begin{array}{c}\text { Experiment and } \\
\text { stimuli }\end{array}$ & $\begin{array}{c}\text { Distances of } \\
0 \text { and 1 }\end{array}$ & $\begin{array}{c}\text { Distances of } \\
1 \text { and 2 }\end{array}$ & $\begin{array}{c}\text { Response } \\
\text { compatibility }\end{array}$ \\
\hline $\begin{array}{l}\text { Experiment 1 } \\
\text { Arabic-Arabic }\end{array}$ & 9 & 15 & 25 \\
Experiment 2 & & & \\
$\quad$ Arabic-words & 4 & 11 & 25 \\
$\quad$ Words-Arabic & 7 & 3 & 27 \\
Experiment 3 & & 16 & 31 \\
$\quad$ Bilateral & 9 & 11 & 29 \\
LVF & 15 & 18 & 30 \\
RVF & 14 & & \\
\hline
\end{tabular}

Note. Mean differences in reaction time (in milliseconds) between distance of 0 and distance of 1 (compatible trials only), between distance of 1 and distance of 2 (compatible trials only), and between incompatible and compatible trials (Experiment 1: Arabic targets and Arabic distractors; Experiment 2: Arabic targets and word distractors or word targets and Arabic distractors; Experiment 3: bilateral, left visual field [LVF], and right visual field $[\mathrm{RVF}]$ conditions with Arabic targets and Arabic distractors). 
agreement with the internal order of representations. This interpretation is also supported by an unpublished number comparison study we conducted recently in which we found that the digits 3 and 4 returned an RVF advantage when the stimulus set consisted of the stimuli $1,2,3$, and 4 but yielded an LVF advantage when the stimulus set consisted of $3,4,5$, and 6 .

\section{Experiment 2}

To further test whether the distance-priming effect in Experiment 1 was due to semantic interactions rather than to perceptual factors, we conducted a second experiment in which target and distractor were of different modalities. Both target and distractor could be either an Arabic digit or a word numeral. (A pilot study in which the target always was an Arabic digit and the distractor always a word numeral did not elicit significant bilateral field interactions, probably because attention could easily be disengaged from the word distractor on the basis of the consistent difference in physical magnitude.) Arabic digits and word numerals belong to different lexicons, as demonstrated by intracranial recordings (Allison, McCarthy, Nobre, Puce, \& Belger, 1994), which indicate that the identification of words and digits activate different but neighboring brain areas.

\section{Method}

Participants. Sixteen undergraduates from Ghent University participated for course credits. All participants (age range = 18-29 years) were men and were right-handed as confirmed by a Dutch translation of the Oldfield (1971) questionnaire. Participants were unaware of the purpose of the experiment and had normal or corrected-to-normal vision.

Procedure. The same apparatus, presentation procedures, and instructions were used as in Experiment 1, except that target and distractor were of different modalities (i.e., when the target was an Arabic digit, the distractor was a word numeral, and vice versa).

Stimuli were Arabic digits 1, 2, 3, 5, 6, and 7 (see Experiment 1 for details) or the Dutch word equivalents een, twee, drie, vijf, zes, and zeven, printed in lowercase. The words were presented horizontally and extended $1^{\circ}$ to $1.7^{\circ}$ horizontally and $0.5^{\circ}$ vertically. Both word targets and distractors were presented at $0.9^{\circ}$ left or right of the fixation mark (measured from the vertical line of the fixation mark to the nearest edge of the first [RVF] or the last [LVF] letter of the word).

Each participant completed a training block of 144 trials and five experimental blocks of 288 trials. This resulted in a total of 10 observations per participant in each of the $2 \times 2 \times 6 \times 6$ $($ Modality $\times \mathrm{VHF} \times$ Target Value $\times$ Distractor Value $)=144$ repeated measures conditions. At the end of each block the participants received feedback about their RTs and accuracy.

\section{Results}

Only average RTs of correct responses in the range of $150-1,500 \mathrm{~ms}$ were analyzed. Error rate did not exceed $10 \%$ per participant $(M=5 \%)$. In addition, the percentage of outliers, averaged across participants, amounted to only $0.2 \%$ of the correct responses. There was no speed-accuracy trade-off, as indicated by a positive correlation between RT and number of errors over the 144 cells of the design $(r=.68, n=144, p<.05)$.
ANOVA. Two separate $2 \times 6 \times 6$ ANOVAs for each target modality (Arabic digits vs. word numerals) were performed with the following repeated measures: VHF (2 levels) and target and distractor values (6 levels each).

Arabic targets. The main effect of VHF was not significant $(\mathrm{LVF}=601 \mathrm{~ms}, \mathrm{RVF}=589 \mathrm{~ms}), F(1,15)=2.62$, $M S E=16,158.92, p=.13$.

There was a significant main effect of target value $(1=$ $577 \mathrm{~ms}, 2=580 \mathrm{~ms}, .3=602 \mathrm{~ms}, 5=619 \mathrm{~ms}, 6=600$ $\mathrm{ms}, 7=590 \mathrm{~ms}), F(5,75)=10.42, M S E=4,419.92, p<$ $.01)$ due to the numerical distance effect (Moyer \& Landauer, 1967): The RTs to target value 1 were significantly faster than the RTs to the values 3,5 , and 6 , as revealed by post hoc comparisons (Tukey $p<.05$ ). The RTs to the Arabic digit 7 were solely faster than the RTs to target value 5 (Tukey $p<.01$ ).

The main effect of distractor value was also significant $(1=581 \mathrm{~ms}, 2=589 \mathrm{~ms}, 3=596 \mathrm{~ms}, 5=603 \mathrm{~ms}, 6=$ $604 \mathrm{~ms}, 7=595 \mathrm{~ms}), F(5,75)=6.09, M S E=2,441.44$, $p<.01$. Post hoc comparisons showed that RTs with distractor value 1 were significantly faster than with 3,5 , and 6 (Tukey $p<.05$ ). RTs with distractor value 2 were significantly faster than with distractor value 6 (Tukey $p<$ $.05)$.

Significant interactions between target and distractor value were observed, $F(25,375)=5.45, M S E=1,982.61$, $p<.01$, indicating interhemispheric interactions (see Table 3 for averaged RTs). Below we examine the origin of this interaction (see semantic priming and response compatibility effect).

There was also a significant interaction between target and VHF, $F(5,75)=2.83, M S E=1,792.51, p<.03($ see Figure 2). For small digits (1, 2, and 3) no VHF effect was present $(\mathrm{LVF}=589 \mathrm{~ms}$ vs. $\mathrm{RVF}=584 \mathrm{~ms})$, whereas for larger digits (5, 6, and 7) an RVF advantage emerged $(\mathrm{LVF}=612 \mathrm{~ms}$ vs. RVF $=593 \mathrm{~ms})$. The RVF advantage was only significant for target values 5 and 6 (Tukey $p<$ $.04)$.

To test response compatibility, we performed a $2 \times 2$ ANOVA with VHF (2 levels) and response compatibility (2 levels) as repeated measures. The main effect of VHF was not significant, $F(1,15)=2.76, M S E=900.21, p=.12$, which repeats the zero effect in the previous ANOVA. Response compatibility was significant, $F(1,15)=17.84$, $M S E=522.72, p<.01$, showing significantly faster RTs in the congruent condition (582 $\mathrm{ms}$ ) than in the incongruent condition (607 ms; see Table 2). Finally, the interaction between $\mathrm{VHF}$ and response compatibility was marginally significant, $F(1,15)=3.24, M S E=163.32, p=.09$, revealing a tendency toward a stronger RVF advantage in the incongruent response condition $(19 \mathrm{~ms})$ than in the congruent condition ( $8 \mathrm{~ms})$.

To test semantic priming, we carried out a $2 \times 3$ ANOVA with VHF (2 levels) and distance between target and distractor (3 levels: distance of 0,1 , and 2) as repeated measures. We included only observations for the targets 1, 3, 5, and 7 in the analysis to have an equivalent distribution of targets in all distance conditions. The main effect of VHF was not significant $(F<1)$. Distance was significant, 
Table 3

Mean Reaction Times in Milliseconds as a Function of Target and Distractor

(Experiment 2: Arabic Targets and Word Distractors or Word Targets and Arabic Distractors)

\begin{tabular}{lcccrrr}
\hline & \multicolumn{7}{c}{ Distractor } \\
\cline { 2 - 6 } Target & een & twee & drie & vijf & zes & zeven \\
\hline 1 & $549(2)$ & $546(2)$ & $576(2)$ & $594(3)$ & $603(4)$ & $596(5)$ \\
2 & $561(2)$ & $551(1)$ & $576(2)$ & $592(3)$ & $613(4)$ & $585(6)$ \\
3 & $591(3)$ & $595(3)$ & $571(7)$ & $617(6)$ & $636(6)$ & $602(5)$ \\
5 & $604(7)$ & $628(4)$ & $628(7)$ & $619(5)$ & $608(3)$ & $624(6)$ \\
6 & $584(5)$ & $611(6)$ & $621(7)$ & $610(3)$ & $584(5)$ & $588(2)$ \\
7 & $596(5)$ & $603(3)$ & $602(5)$ & $585(2)$ & $580(4)$ & $574(2)$ \\
\hline & 1 & 2 & 3 & 5 & 6 & 7 \\
een & $578(1)$ & $583(2)$ & $577(1)$ & $612(4)$ & $619(5)$ & $619(7)$ \\
twee & $627(4)$ & $618(3)$ & $633(4)$ & $654(11)$ & $666(10)$ & $667(10)$ \\
drie & $647(8)$ & $650(7)$ & $628(4)$ & $665(10)$ & $682(13)$ & $674(9)$ \\
vijf & $620(4)$ & $628(8)$ & $647(8)$ & $601(4)$ & $625(4)$ & $617(4)$ \\
zes & $625(10)$ & $637(8)$ & $645(7)$ & $624(4)$ & $605(3)$ & $624(5)$ \\
zeven & $614(6)$ & $637(3)$ & $641(4)$ & $618(3)$ & $589(3)$ & $611(6)$ \\
\hline
\end{tabular}

Note. Values in parentheses are percentages of errors.

$F(2,30)=4.35, M S E=454.80, p<.05$ (see Figure 3 ), showing slower RTs with increasing distance between target and distractor (distance of $0=578 \mathrm{~ms}$; distance of $1=$ $582 \mathrm{~ms}$; distance of $2=593 \mathrm{~ms}$; see Table 2). Planned comparisons revealed a significant difference between distance of 0 and distance of $2(p<.01)$, whereas the difference between distance of 1 and distance of 2 was only marginally signicant $(p=.06)$. Finally, the interaction between VHF and distance was not significant $(F<1)$.

Word targets. The main effect of VHF was marginally significant $(\mathrm{LVF}=637 \mathrm{~ms}, \mathrm{RVF}=619 \mathrm{~ms}), \quad F(1$, $15)=3.32, M S E=27,999.43, p=.09$.

There was a significant main effect of target value $(1=$ $598 \mathrm{~ms}, 2=644 \mathrm{~ms}, 3=658 \mathrm{~ms}, 5=623 \mathrm{~ms}, 6=627 \mathrm{~ms}$, $7=618 \mathrm{~ms}), F(5,75)=13.45, M S E=6,178.97, p<.01$.

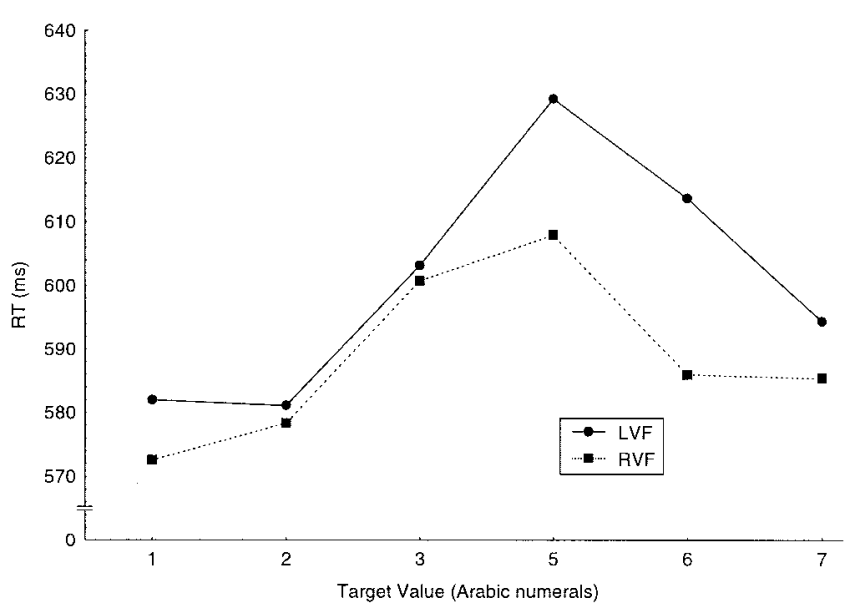

Figure 2. Significant interaction between visual half field and target value in Experiment 2 in the Arabic target condition. RT = reaction time; $\mathrm{LVF}=$ left visual field; $\mathrm{RVF}=$ right visual field.
The RTs to the word een were significantly faster than to twee, drie, vijf, and zes, as revealed by post hoc comparisons (Tukey $p<.03$ ). This effect may be ascribed to the higher frequency of the word een in Dutch (een is also the indefinite article $a(\mathrm{n})$ in Dutch) and to the numerical distance effect (Moyer \& Landauer, 1967).

Significant interactions between target and distractor value were observed, $F(25,375)=6.72, M S E=1,748.45$, $p<.01$, indicating interhemispheric interactions (see Table 3 for averaged RTs).

To test response compatibility, we performed a $2 \times 2$ ANOVA with VHF ( 2 levels) and response compatibility (2 levels) as repeated measures. The main effect of VHF was marginally significant, $F(1,15)=3.51, M S E=1,503.23$,

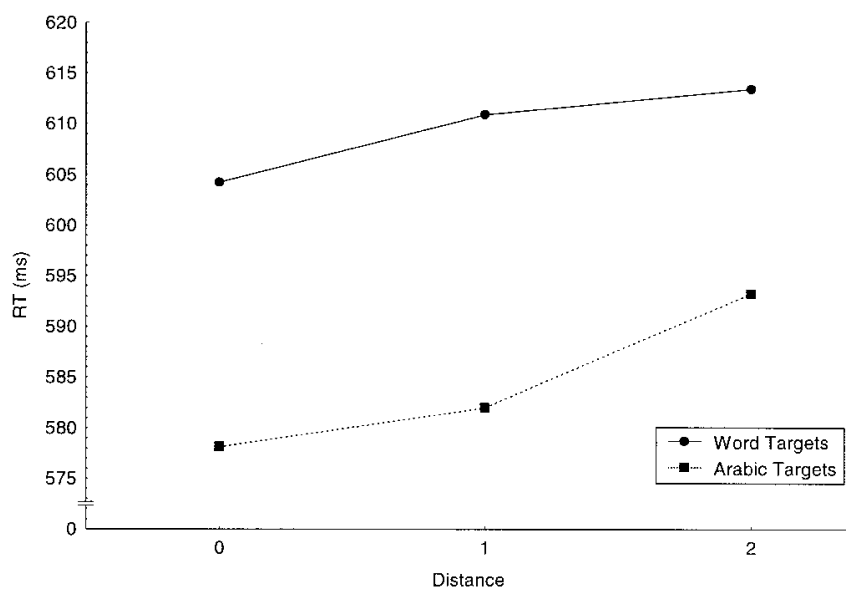

Figure 3. Significant distance effects between target and distractor in Experiment 2 in which target and distractor were of different modalities (Arabic targets vs. word distractors and word targets vs. Arabic distractors). $\mathrm{RT}=$ reaction time. 
$p=.08$, showing faster RTs in the RVF (see also previous analysis). Response compatibility was significant, $F(1,15)$ $=54.87, M S E=221.02, p<.05$, showing significantly faster RTs in the congruent condition (614 ms) than in the incongruent condition (641 ms, see Table 2). Finally, the interaction between VHF and response compatibility was not significant $(F<1)$.

To test semantic priming we carried out a $2 \times 3$ ANOVA with VHF ( 2 levels) and distance between target and distractor (3 levels: distance of 0,1 , and 2) as repeated measures. We included only observations for the targets $1,3,5$, and 7 in the analysis to have an equivalent distribution of targets in all distance conditions. The main effect of VHF was significant, $F(1,15)=4.77, M S E=2,056.47, p=.05$, showing an RVF advantage $(\mathrm{LVF}=620 \mathrm{~ms}, \mathrm{RVF}=599$ $\mathrm{ms}$ ). Distance did not reach significance, $F=1.2$ (distance of $0=604 \mathrm{~ms}$, distance of $1=611 \mathrm{~ms}$, distance of $2=614$ $\mathrm{ms}$; see Figure 3 and Table 2), and did not interact with VHF $(F<1)$. To test whether the absence of a significant distance effect was due to the fact that the analysis was limited to the targets $1,3,5$, and 7 , we ran another analysis in which all target values $(1,2,3,5,6$, and 7$)$ were included. Only distance of 0 and distance of 1 were included in the analysis because targets 2 and 6 did not have distractors with distance of 2 . In this new analysis with more observations and more power, the effect of distance turned out to be significant, $F(1,15)=8.94, M S E=264.11, p<.01$, showing faster RTs when target and distractor were of the same value (distance of $0=607 \mathrm{~ms}$, distance of $1=619$ $\mathrm{ms})$.

\section{Discussion}

Even when target and distractor belong to a different mental lexicon (Arabic digits vs. word numerals), significant interhemispheric interactions are observed. The bilateral field interactions not only revealed significant response compatibility effects but also revealed significant-albeit smaller-distance effects (see Table 2). This is further evidence for our hypothesis that the interhemispheric interactions are largely semantic in nature and cannot be attributed to perceptual priming. The fact that the distance effect was smaller in Experiment 2 than in Experiment 1 is probably due to the fact that the difference in physical size between the verbal and the Arabic stimuli provided the participants with the opportunity to direct their attention more rapidly to the target and to discard the distractor. In line with Ratinckx et al. (2001), there was no evidence for strong VHF asymmetries. Only for word targets did we obtain some indication of a superiority of the LH. There was an interaction between target value and VHF, but as mentioned in the Discussion section of Experiment 1, we believe this has more to do with the hypothesized left-right orientation of the semantic number line than with hemispheric differences in the processing of large and small numbers.

\section{Experiment 3}

To further evaluate the strength of the interhemispheric interactions in number comparison, we designed an addi- tional experiment in which the bilateral Stroop-like task was compared with a unilateral one (both target and distractor were again Arabic numerals, as in Experiment 1). Target and distractor were presented either in different VHFs or in the same VHF. If the stimulus presentation is properly controlled, such a design allows us to directly compare the effects of distance and response compatibility when the stimuli are presented bilaterally, unilaterally in LVF, and unilaterally in RVF. On the basis of our previous findings, we did not expect large differences between the bilateral and the unilateral presentation conditions or between the LVF and the RVF unilateral presentation conditions. Additionally, we added a neutral condition to the design in which the distractor was not a number but a \# sign, so that we could examine to what extent the distance effect was due to interference or facilitation by the distractor on the processing of the target.

\section{Method}

Participants. Twenty-four volunteers were paid $\$ 15$ to participate in the experiment. All participants (age range $=18-38$ years) were men and were right-handed as confirmed by a Dutch translation of the Oldfield (1971) questionnaire. Participants were unaware of the purpose of the experiment and had normal or corrected-to-normal vision.

Procedure. The same apparatus, presentation procedures, and instructions were used as in Experiments 1 and 2, except that the stimulus display now could be one of four possible VHF conditions (see Figure 4), that is, two unilateral (LVF/RH or RVF/LH) and two bilateral conditions. In the unilateral conditions target and

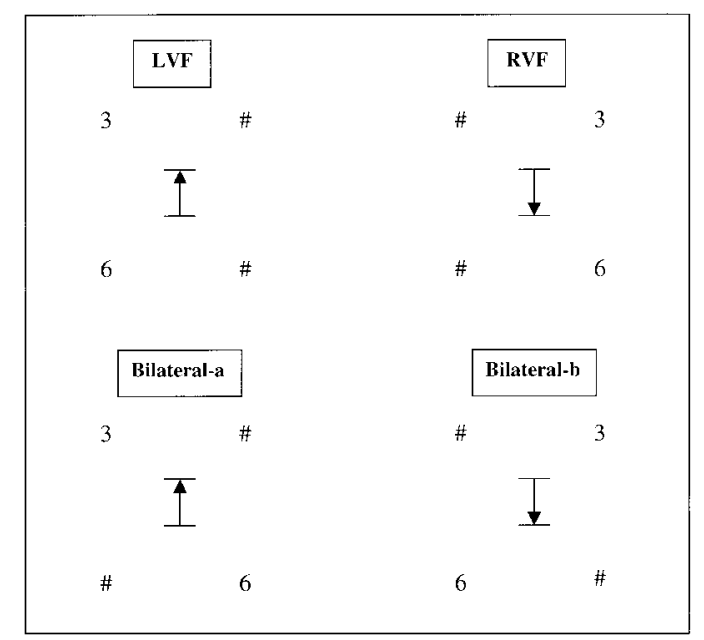

Figure 4. The four visual half field conditions in Experiment 3. The intrahemispheric condition consisted of the two upper displays left visual field (LVF)/right hemisphere and right visual field (RVF)/left hemisphere; the two lower displays belonged to the interhemispheric condition (Bilateral-a, target and distractor in the upper left corner and lower right corner; Bilateral-b, target and distractor in the lower left corner and upper right corner). In the neutral condition, the distractor was replaced by a \# sign. The arrow in the fixation location could point either upward or downward to the target number. 
distractor were presented randomly one above the other in the LVF $(\mathrm{RH})$ or in the RVF (LH), whereas in the bilateral conditions, target and distractor were presented diagonally in opposite visual fields. To equate the perceptual load directed to each hemisphere on within- and across-hemisphere trials we made use of noise stimuli, using the \# sign (see Marks \& Hellige, 1999, for the same design). In the bilateral trials, the target was also presented randomly in the upper or the lower position, resulting in two diagonal conditions per VHF (see Figure 4). Because the bilateral conditions consisted of diagonal presentation of target and distractor, possible left-right scanning effects related to reading were reduced as much as possible (see, e.g., Banich \& Schenker, 1994; Marks \& Hellige, 1999).

The fixation stimulus consisted of two short horizontal lines of $0.2^{\circ}$, one above the other, separated by a gap of $0.5^{\circ}$. An arrow was presented in the fixation location pointing upward or downward to the target number. A stimulus display consisted of 2 noise stimuli (\# sign) and a target and distractor stimulus, which were presented at the corners of an imaginary rectangle around the fixation location (see Figure 4). The width of the imaginary rectangle was $1.8^{\circ}$, the length $2.5^{\circ}$. This resulted in an eccentricity of $1.5^{\circ}$ (measured from the center of the stimulus to the center of the fixation location). The distance between target and distractor in the unilateral condition was $2.5^{\circ}$, in the bilateral condition $3.1^{\circ}$ (it was not possible to completely equate these distances because of trigonometrical constraints; see Banich \& Schenker, 1994). In the neutral condition the distractor number was replaced by a \# sign. Stimuli were the Arabic numerals 1, 2, 3, 5, 6, and 7 and the noise (neutral) stimulus \#, extending $0.3^{\circ} \times 0.4 \times$ horizontally and vertically.

In two separate sessions of $1.5 \mathrm{hr}$ each, participants completed one training block including all experimental conditions and 11 experimental blocks of 336 trials. This resulted in a total of 11 observations per participant in each of the $4 \times 2 \times 6 \times 7$ (VHF $\times$ Target Position $\times$ Target Value $\times$ Distractor Value $)=336$ repeated measures conditions. Participants simply had to indicate whether the number in the upper or the lower part of the figure (depending on where the arrow pointed) was smaller or larger than 4 . They were told to ignore the other number (if present). At the end of each block the participants received feedback about their mean RT and accuracy.

\section{Results}

Only average RTs of correct responses in the range of $150-1,500 \mathrm{~ms}$ were analyzed. Error rate did not exceed $15 \%$ per participant $(M=7.1 \%)$. In addition, the percentage of outliers, averaged across participants, amounted to only $0.1 \%$ of the correct responses. There was no speed-accuracy trade-off, as indicated by a positive correlation between RT and number of errors over the 336 cells of the design $(r=.76, n=336, p<.05)$.

ANOVA. Two separate $2 \times 6 \times 6$ ANOVAs were performed, one for the intrahemispheric conditions and one for the interhemispheric conditions. The following repeated measures were included in the analysis: VHF (2 levels: LVF and RVF), target value (6 levels), and distractor value (7 levels, including the neutral condition). For each condition we collapsed the data across the upper and the lower positions of the target.

Intrahemispheric conditions. The main effect of VHF was not significant $(\mathrm{LVF}=543 \mathrm{~ms}, \mathrm{RVF}=540 \mathrm{~ms}), F(1$, 23) $=1.95, M S E=1,323.44, p=.18$.
There was a main effect of target value $(1=528 \mathrm{~ms}, 2=$ $542 \mathrm{~ms}, 3=565 \mathrm{~ms}, 5=550 \mathrm{~ms}, 6=535 \mathrm{~ms}, 7=529 \mathrm{~ms})$, $F(5,115)=20.27, M S E=3,279.39, p<.01$, due to the numerical distance effect (Moyer \& Landauer, 1967). Post hoc comparisons revealed that RTs to target 1 were significantly faster than to 2 and 5 (Tukey $p<.04$ ). RTs to target 3 were significantly slower than to all other target values (Tukey $p<.02$ ), and RTs to target 5 were also significantly slower than to 6 and 7 (Tukey $p<.02$ ).

The main effect of distractor value was also significant $(1=543 \mathrm{~ms}, 2=540 \mathrm{~ms}, 3=538 \mathrm{~ms}, 5=548 \mathrm{~ms}, 6=$ $547 \mathrm{~ms}, 7=557 \mathrm{~ms}$, neutral condition $=517 \mathrm{~ms}), F(6$, $138)=44.68, M S E=984.62, p<.01$. Post hoc comparisons revealed that RTs in the neutral condition were significantly faster than RTs with all distractor numbers (Tukey $p<.01$ ). In addition, RTs with distractor 7 were significantly slower than with all other values (Tukey $p<$ $.05)$, RTs with distractor 6 were significantly slower than with value 3 (Tukey $p<.05$ ), and RTs with distractor 5 were significantly slower than with 2 and 3 (Tukey $p<.05$ ).

Significant interactions between target and distractor value were observed, $F(30,690)=26.14, M S E=640.75$, $p<.01$, indicating intrahemispheric interactions (see Table 4 for averaged RTs).

To test response compatibility, we performed a $2 \times 2$ ANOVA with VHF ( 2 levels) and response compatibility (2 levels) as repeated measures. Only the response compatibility effect was significant, $F(1,23)=89.40, M S E=$ 227.13, $p<.01$ (see Figure 5 and Table 2), showing faster RTs in the congruent condition $(531 \mathrm{~ms})$ than in the incongruent condition $(560 \mathrm{~ms})$.

To test distance priming, we carried out a $2 \times 4$ ANOVA with VHF (2 levels) and distance between target and distractor (4 levels: neutral condition, distance of 0,1 , or 2 ) as repeated measures. We included only observations for the targets $1,3,5$, and 7 in the analysis to have an equivalent distribution of targets in all distance conditions. Distance was the only significant effect, showing slower RTs with increasing distance between target and distractor, $F(3$, 69 ) $=45.35, M S E=183.68, p<.01$ (see Figure 6 and Table 2). The RTs with the neutral distractor did not differ significantly from the condition in which target and distractor were the same numeral (neutral condition $=519 \mathrm{~ms}$, distance of $0=517 \mathrm{~ms}$, distance of $1=531 \mathrm{~ms}$, distance of $2=546 \mathrm{~ms}$ ). Planned comparisons revealed that both distance of 0 and the neutral condition differed significantly from distances of 1 and $2(p<.01)$. Distance of 1 also differed significantly from distance of 2 , indicating semantic priming $(p<.01)$. To investigate the presence of perceptual priming, we tested whether there was evidence for a quadratic component in addition to a linear component in the distance effect (see Experiment 1). The linear trend was significant, $F(1,23)=92.13, M S E=212.53, p<.01$, but the quadratic trend was not $(F<1)$. The interaction between VHF and distance was not significant either $(F<1)$.

Interhemispheric conditions. The main effect of VHF was not significant $(\mathrm{LVF}=540 \mathrm{~ms}, \mathrm{RVF}=538 \mathrm{~ms} ; F<$ 1). There was a main effect of target value $(1=528 \mathrm{~ms}, 2=$ $536 \mathrm{~ms}, 3=557 \mathrm{~ms}, 5=549 \mathrm{~ms}, 6=536 \mathrm{~ms}, 7=529 \mathrm{~ms}$ ), 
Table 4

Mean Reaction Times in Milliseconds as a Function of Target and Distractor

(Experiment 3: Intra- and Interhemispheric Conditions With Arabic Numerals as Targets and Distractors)

\begin{tabular}{|c|c|c|c|c|c|c|c|}
\hline \multirow[b]{2}{*}{ Target } & \multicolumn{7}{|c|}{ Distractor } \\
\hline & 1 & 2 & 3 & 5 & 6 & 7 & $\#$ \\
\hline \multicolumn{8}{|c|}{ Intrahemispheric condition } \\
\hline $\begin{array}{l}1 \\
2 \\
3 \\
5 \\
6 \\
7\end{array}$ & $\begin{array}{l}496(4) \\
544(9) \\
568(10) \\
558(4) \\
548(9) \\
543(11)\end{array}$ & $\begin{array}{l}513(4) \\
512(9) \\
548(7) \\
566(5) \\
551(10) \\
549(8)\end{array}$ & $\begin{array}{l}521(7) \\
527(11) \\
534(4) \\
563(10) \\
549(14) \\
537(7)\end{array}$ & $\begin{array}{l}543(4) \\
560(8) \\
583(5) \\
535(6) \\
535(10) \\
534(8)\end{array}$ & $\begin{array}{l}550(3) \\
565(10) \\
587(7) \\
542(3) \\
511(16) \\
525(12)\end{array}$ & $\begin{array}{l}564(7) \\
569(7) \\
594(5) \\
564(9) \\
542(9) \\
508(5)\end{array}$ & $\begin{array}{l}509(6) \\
513(7) \\
539(5) \\
523(7) \\
511(9) \\
507(6)\end{array}$ \\
\hline \multicolumn{8}{|c|}{ Interhemispheric condition } \\
\hline $\begin{array}{l}1 \\
2 \\
3 \\
5 \\
6 \\
7\end{array}$ & $\begin{array}{l}496(4) \\
537(4) \\
562(11) \\
564(10) \\
558(10) \\
543(9)\end{array}$ & $\begin{array}{l}516(3) \\
509(4) \\
539(8) \\
570(11) \\
557(8) \\
552(10)\end{array}$ & $\begin{array}{l}517(3) \\
521(3) \\
527(6) \\
555(10) \\
548(9) \\
533(7)\end{array}$ & $\begin{array}{l}546(7) \\
550(7) \\
573(10) \\
531(7) \\
528(5) \\
531(4)\end{array}$ & $\begin{array}{l}552(7) \\
556(8) \\
581(10) \\
535(9) \\
512(5) \\
519(5)\end{array}$ & $\begin{array}{l}556(7) \\
561(7) \\
579(11) \\
563(8) \\
534(5) \\
515(3)\end{array}$ & $\begin{array}{l}511(4) \\
518(5) \\
535(8) \\
527(6) \\
512(5) \\
514(4)\end{array}$ \\
\hline
\end{tabular}

Note. Values in parentheses are percentages of errors. In the neutral condition, the \# sign was used as a distractor.

$F(5,115)=16.27, M S E=2,718.71, p<.01$, due to the numerical distance effect (Moyer \& Landauer, 1967). Post hoc comparisons revealed that RTs to targets 3 and 5 were significantly slower than to $1,2,6$, and 7 (Tukey $p<.03$ ).

The main effect of distractor value was also significant $(1=543 \mathrm{~ms}, 2=540 \mathrm{~ms}, 3=533 \mathrm{~ms}, 5=543 \mathrm{~ms}, 6=$ $542 \mathrm{~ms}, 7=551 \mathrm{~ms}$, neutral condition $=519 \mathrm{~ms}), F(6$, $138)=33.28, M S E=879.17, p<.01$. Post hoc comparisons revealed that RTs in the neutral condition were significantly faster than RTs with all distractor numbers (Tukey $p<.01$ ). In addition RTs with distractor 7 were significantly slower than with all other values (Tukey $p<$

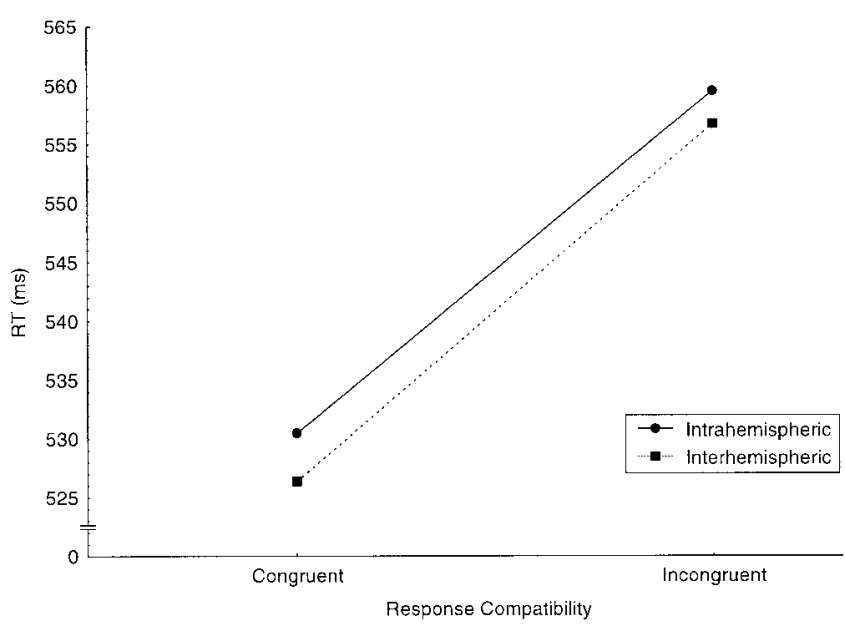

Figure 5. Nonsignificant interaction between response compatibility and stimulus display in Experiment 3, indicating strong interhemispheric integration of the response preparation system. $\mathrm{RT}=$ reaction time.
.02) and RTs with distractors 1,5 , and 6 were significantly slower than with value 3 (Tukey $p<.05$ ).

Significant interactions between target and distractor value were observed, $F(30,690)=28.46, M S E=599.66$, $p<.01$, indicating interhemispheric interactions (see Table 4 for averaged RTs).

To test response compatibility, we performed a $2 \times 2$ ANOVA with VHF (2 levels) and response compatibility (2 levels) as repeated measures. Only the response compatibility effect was significant, $F(1,23)=104.85, M S E=$ 210.94, $p<.01$ (see Figure 5 and Table 2), showing faster RTs in the congruent condition $(526 \mathrm{~ms})$ than in the incongruent condition (557 ms).

To test distance priming, we carried out a $2 \times 4$ ANOVA with VHF (2 levels) and distance between target and distractor (4 levels: neutral condition, distance of 0,1 , or 2 ) as repeated measures. As in all previous analyses, we included only observations for the targets $1,3,5$, and 7 to obtain an equivalent distribution of targets in all distance conditions. Only the main effect of distance was significant, because of slower RTs with increasing distance between target and distractor, $F(3,69)=34.95, M S E=165.67, p<.01$ (neutral condition $=521 \mathrm{~ms}$, distance of $0=517 \mathrm{~ms}$, distance of $1=526 \mathrm{~ms}$, distance of $2=542 \mathrm{~ms}$; see Figure 6 and Table 2). Planned comparisons revealed that the difference between distance of 0 and the neutral condition was marginally significant $(p=.07)$, showing faster RTs when target and distractor were the same numeral. The difference between distance 1 and the neutral condition was also marginally significant ( $p=.07$ ), showing faster RTs in the neutral condition. Distance of 0 differed significantly from distance of 1 and distance of $2(p=.01)$, whereas the neutral condition differed significantly from distance of 2 only $(p=.01)$. Finally, distance of 1 differed significantly 


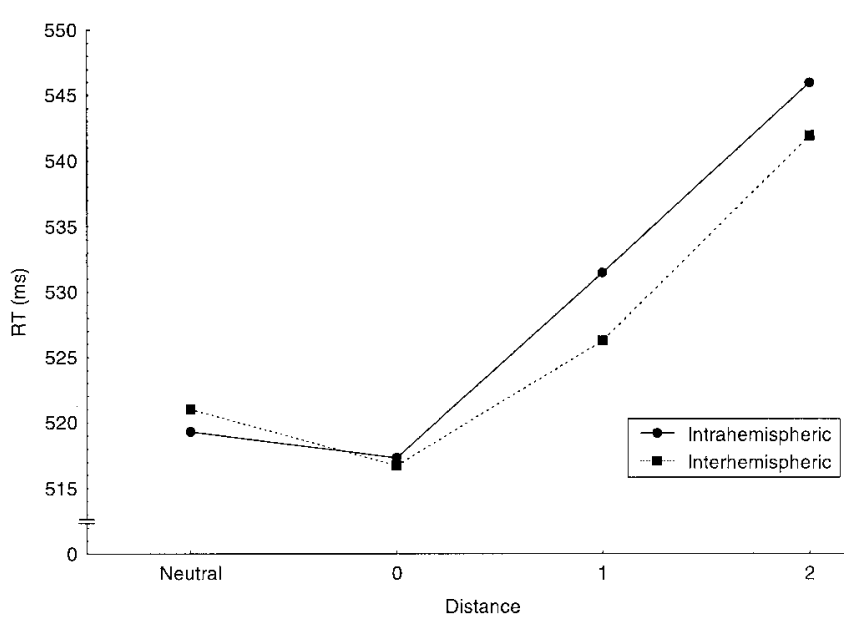

Figure 6. Nonsignificant interaction between distance and stimulus display in Experiment 3, indicating strong bilateral integration of the abstract magnitude system. $\mathrm{RT}=$ reaction time.

from distance of 2 , indicating semantic priming $(p<.01)$. To investigate the presence of perceptual priming, we tested whether there was a quadratic component in addition to a linear component in the distance effect. The linear trend was significant, $F(1,23)=106.93, M S E=141.74, p<.01$, but the quadratic trend did not reach significance, $F(1$, 23) $=2.02, M S E=149.74, p=.17$.

The interaction between VHF and distance was significant (LVF: neutral condition $=522 \mathrm{~ms}$, distance of $0=516$ $\mathrm{ms}$, distance of $1=532 \mathrm{~ms}$, distance of $2=540 \mathrm{~ms}$; RVF: neutral condition $=520 \mathrm{~ms}$, distance of $0=517 \mathrm{~ms}$, distance of $1=521 \mathrm{~ms}$, distance of $2=543 \mathrm{~ms}), F(3$, $69)=3.83, M S E=115.64, p<.05$. Planned comparisons showed that RTs differed at only distance of 1 , where there was an RVF advantage $(p<.05)$.

Intra- vs. interhemispheric conditions. To investigate whether distance priming and response compatibility differed between interhemispheric and intrahemispheric processing we run two additional ANOVAs.

To test differences in response compatibility, we performed a $2 \times 2$ ANOVA with stimulus display ( 2 levels: bilateral vs. unilateral) and response compatibility (2 levels) as repeated measures. The main effect of stimulus display was significant, revealing faster RTs with bilateral stimulus displays than with unilateral displays (542 ms vs. $545 \mathrm{~ms}$ ), $F(1,23)=18.82, M S E=15.20, p<.05$. As in all previous analyses, response compatibility, $F(1,23)=105.07$, $M S E=201.78, p<.01$, was significant, revealing faster RTs in the congruent condition ( $528 \mathrm{~ms}$ vs. $558 \mathrm{~ms})$. Finally, the interaction between stimulus display and response compatibility failed to reach significance $(F<1$; see Figure 5 and Table 2).

To test differences in distance priming, we performed a $2 \times 4$ ANOVA with stimulus display (2 levels: bilateral vs. unilateral) and distance between target and distractor (4 levels, including the neutral condition) as repeated measures factors. As in all previous analyses, we included only ob- servations for the targets $1,3,5$, and 7 to obtain an equivalent distribution of targets in all distance conditions. The main effect of stimulus display was significant, revealing faster RTs after bilateral displays (527 ms vs. $529 \mathrm{~ms}), F(1$, $23)=5.22, M S E=38.32, p<.05$. As in all previous analyses the main effect of distance was significant, showing slower RTs with increasing distance between target and distractor, $F(3,69)=65.01, M S E=106.75, p<.01$ (neutral condition $=520 \mathrm{~ms}$, distance of $0=517 \mathrm{~ms}$, distance of $1=529 \mathrm{~ms}$, distance of $2=544 \mathrm{~ms}$ ). Planned comparisons revealed that both distance of 0 and the neutral condition differed significantly from distance of 1 and 2 $(p<.05)$. Distance of 1 also differed significantly from distance of 2 , indicating semantic priming $(p<.01)$. The difference between the neutral condition and distance of 0 was marginally significant, revealing a tendency of faster RTs in distance of $0(p=.07)$. To investigate the presence of perceptual priming, we tested whether there was evidence for a quadratic component in addition to a linear component in the distance effect. The linear trend was significant, $F(1$, $23)=145.15, M S E=119.17, p<.01$, whereas the quadratic trend did not reach significance, $F(1,23)=1.02$, $M S E=82.65, p=.32$. Finally, the interaction between distance and stimulus display failed to reach significance, $F(2,46)=1.21, M S E=57.19, p=.31$ (see Figure 6 and Table 2).

\section{Discussion}

As expected from previous research (e.g., Boles, 1986; Ratinckx et al., 2001), no hemispheric differences were observed in the number comparison task. Neither in the intrahemispheric nor in the interhemispheric conditions did we observe significant differences between LVF and RVF. There was no main effect of VHF, Moyer and Landauer's (1967) distance effect was the same for both VHFs (see also Cohen \& Dehaene, 1996), and the distance effect of the distractor on the processing of the target was the same in LVF and RVF.

The effect of the distractor on the processing of the target was largely based on interference, as RTs were slower in the conditions with two different digits than in the conditions with a neutral distractor. Only when target and distractor were the same (distance of 0 ), was there some evidence for facilitation ( $3 \mathrm{~ms} ; p=.07$ ), compared with the neutral condition. Overall, these findings are in line with Boles's (1995) hypothesis that bilateral stimulus displays increase the size of VHF asymmetries (both LVF and RVF advantages) because of the interhemispheric competition induced by the two stimuli.

As in all previous experiments, rich interhemispheric interactions were observed. First, both the distance and the response compatibility effect of Experiment 1 were replicated (see the interhemispheric conditions). A look at Table 2 shows the remarkable similarity of the magnitudes of the effects in both experiments, despite the facts that (a) the distance between target and distractor was smaller in Experiment $1\left(2.8^{\circ}\right)$ than in Experiment $3\left(3.1^{\circ}\right)$ and (b) both stimuli were presented horizontally in Experiment 1 but 
diagonally in Experiment 3. The only difference between the experiments was the significant interaction between distance and VHF in Experiment 3. As this interaction was due to one single deviating data pair (at distance of 1) and was not present in Experiment 1 (or in Ratinckx et al., 2001), we think it is better at present not to attach great importance to this observation.

A comparison of the interhemispheric conditions with the intrahemispheric conditions in the first place showed the remarkable similarity between both types of conditions (Table 2): The response compatibility effect and the distance effect were virtually identical (Figures 5 and 6). The only difference was the small but significant main effect of stimulus display: RTs were some $3 \mathrm{~ms}$ faster with bilateral stimulus presentation than with unilateral stimulus presentation. As this difference was not present in the conditions with distance 0 (Figure 6), it looks like the interference effect of the distractor was slightly but consistently smaller in the interhemispheric than in the intrahemispheric situation. However, the small size of this modulation in no way sufficed to wipe out the more robust effects of response compatibility and semantic priming. The lack of a clear difference between the interhemispheric and the intrahemispheric conditions is the more surprising because it has been shown that split-brain patients have no problems comparing digits presented within the same VHF but are at chance level when the stimuli are presented in opposite VHFs (Corballis, 1994; Seymour et al., 1994).

Finally, neither in the interhemispheric nor in the intrahemispheric conditions did we find evidence for perceptual priming. This is in line with Boles's (1986) hypothesis that, in contrast with odd-even judgment or number recognition, magnitude judgment deemphasizes the perception of specific number information, thereby reducing the visuospatial or verbal emphasis of the task (see also Ratinckx et al., 2001).

\section{General Discussion}

The experiments of the present study further explored semantic interhemispheric integration in number comparison by examining interactions between a target and a distractor in an interhemispheric Stroop-like paradigm (see also Kavcic \& Clarke, 2000, for the use of this task). Significant bilateral field interactions in number comparison have been reported before (Ratinckx et al., 2001) but could not distinguish between interhemispheric interactions at the perceptual level and at the level of an abstract quantity representation.

In line with Ratinckx et al. (2001), all experiments revealed clear evidence of interhemispheric interactions as demonstrated by significant bilateral field interactions. In Experiment 1, in which both target and distractor were Arabic digits, a significant bilateral distance effect and response compatibility effect were observed (Table 2). The distance effect seemed to be entirely semantic in nature, as demonstrated by the significant linear trend and the absence of a quadratic component. Further evidence for this hypothesis was obtained in Experiment 2, in which target and distractor belonged to different modalities and were very likely processed in discrete perceptual systems (Allison et al., 1994). In this experiment, too, a significant distance effect and response compatibility effect were observed, providing strong evidence for interhemispheric integration at the semantic and motor stages of number comparison. The fact that the response compatibility effect was the same in the within-modality experiment and the between-modality experiment suggests that the interhemispheric cross-talk giving rise to this effect happened at the stage of a stimulusindependent response preparation system. In such a response system, stimuli that are repeated a number of times and that always require the same response automatically activate an episodic event-file, which contains the action to be performed (see, e.g., Hommel, 1998). The fact that the response compatibility effect is modality independent has been reported before with central stimulus presentation (Naccache \& Dehaene, 2001; Reynvoet, Caessens, \& Brysbaert, in press) and shows that automatic stimulus-response associations can be semantically mediated.

Experiment 3 directly compared intrahemispheric with interhemispheric processing. Overall, RTs were some $3 \mathrm{~ms}$ slower in the intrahemispheric conditions than in the interhemispheric conditions, suggesting that interference was slightly smaller when the stimuli were distributed over both hemispheres. The most striking finding, however, was the similarity of the distance and the response compatibility effects in the interhemispheric and the intrahemispheric conditions (Figures 5 and 6 and Table 2). This again shows how strong the interhemispheric connections are between the semantic number representations in the left and the right cerebral hemisphere (Dehaene, 1992), certainly because it is known that split-brain patients cannot compare numbers presented in opposite VHFs (Corballis, 1994). In addition, our results are in line with the assumption of symmetric representations in the hemispheres, as there were no VHF differences that required an explanation in terms of laterality.

Our experiments clearly showed that the interhemispheric distance effect we observed was situated at the semantic level and not at the perceptual level. In neither the intrahemispheric nor the interhemispheric conditions did we find any evidence of perceptual priming, which is in line with the hypothesis of Boles (1986) that magnitude judgment is only weakly determined by the perception of the specific number information, thereby producing less strong VHF effects than other numerical tasks, such as number recognition or oddeven judgment (see also Ratinckx et al., 2001). This hypothesis predicts weaker perceptual priming in magnitude judgment than in number recognition or odd-even judgment.

Experiment 3 also included a neutral condition in which we replaced the distractor number with a neutral stimulus (the \# sign) to investigate to what extent the effect of the distractor on the processing of the target was due to interference or facilitation. As can be seen in Figure 6, the effect was predominantly due to interference, as RTs were slower when two different digits were presented than when the distractor was a neutral symbol. There was only weak 
evidence for a small facilitation effect when target and distractor were identical. Again, the findings were very similar in the interhemispheric and the intrahemispheric conditions.

In conclusion, our data complement and replicate previous research (Ratinckx et al., 2001) that pointed to an equivalent bilateral representation of number magnitude in the cerebral hemispheres with strong interhemispheric interactions. The present experiments demonstrate that interhemispheric interactions in number comparison are situated at both the semantic and motor levels. The results are in line with Dehaene's (1992) neuroanatomical model of number processing, which assumes that the analogical magnitude representation has a copy in the LH and RH and that both copies are linked via direct transcallosal pathways (see also Cohen \& Dehaene, 1996).

\section{References}

Allison, T., McCarthy, G., Nobre, A., Puce, A., \& Belger, A. (1994). Human extrastriate visual cortex and the perception of faces, words, numbers and colors. Cerebral Cortex, 5, 544-554.

Banich, M. T., \& Belger, A. (1990). Interhemispheric interaction: How do the hemispheres divide and conquer a task? Cortex, 26, 77-91.

Banich, M. T., \& Schenker, J. I. (1994). Investigations of interhemispheric processing: Methodological considerations. Neuropsychology, 8, 263-277.

Boles, D. B. (1986). Hemispheric differences in the judgment of number. Neuropsychologia, 24, 511-519.

Boles, D. B. (1987). Reaction time asymmetry through bilateral versus unilateral stimulus presentation. Brain and Cognition, 6, 321-333.

Boles, D. B. (1990). What bilateral displays do. Brain and Cognition, 12, 205-228.

Boles, D. B. (1995). Parameters of the bilateral effect. In F. L. Kitterle (Ed.), Hemispheric communication: Mechanisms and models (pp. 211-230). Hillsdale, NJ: Erlbaum.

Bovens, N., \& Brysbaert, M. (1990). IBM PC/XT/AT and PS/2 Turbo Pascal timing with extended resolution. Behavior Research Methods, Instruments and Computers, 22, 332-334.

Brysbaert, M. (1990). A warning about millisecond timing in Turbo Pascal. Behavior Research Methods, Instruments and Computers, 56, 479-490.

Brysbaert, M. (1995). Arabic number reading: On the nature of the numerical scale and the origin of phonological recoding. Journal of Experimental Psychology: General, 124, 434-452.

Brysbaert, M., Bovens, N., d'Ydewalle, G., \& Van Calster, J. (1989). Turbo Pascal timing routines for the IBM microcomputer family. Behavior Research Methods, Instruments and Computers, 21, 73-83.

Chochon, F., Cohen, L., van de Moortele, P. F., \& Dehaene, S. (1999). Differential contributions of the left and right inferior parietal lobules to number processing. Journal of Cognitive Neuroscience, 11, 617-630.

Cohen, L., \& Dehaene, S. (1996). Cerebral networks for number processing: Evidence from a case of posterior callosal lesion. Neurocase, 2, 155-173.

Corballis, M. C. (1994). Can commissurotomized subjects compare digits between the visual fields? Neuropsychologia, 32, $1475-1486$.
Dahmen, W., Hartje, W., Buessing, A., \& Sturm, W. (1982). Disorders of calculation in aphasic patients: Spatial and verbal components. Neuropsychologia, 20, 145-153.

Dehaene, S. (1992). Varieties of numerical abilities. Cognition, 44, $1-42$.

Dehaene, S. (1996). The organization of brain activations in number comparison: Event-related potentials and the additive-factors method. Journal of Cognitive Neuroscience, 8, 47-68.

Dehaene, S., Bossini, S., \& Giraux, P. (1993). The mental representation of parity and number magnitude. Journal of Experimental Psychology: General, 122, 371-396.

Dehaene, S., Dehaene-Lambertz, G., \& Cohen, L. (1998). Abstract representations of numbers in the animal and human brain. Trends in Neurosciences, 21, 355-361.

Dehaene, S., Dupoux, E., \& Mehler, J. (1990). Is numerical comparison digital: Analogical and symbolic effects in two-digit number comparison. Journal of Experimental Psychology: Human Perception and Performance, 16, 626-641.

Dehaene, S., Naccache, L., Le Clec'H, G., Koechlin, E., Mueller, M., Dehaene-Lambertz, G., et al. (1998). Imaging unconscious semantic priming. Nature, 395, 597-600.

Fias, W., Brysbaert, M., Geypens, F., \& d'Ydewalle, G. (1996). The importance of magnitude information in numerical processing: Evidence from the SNARC effect. Mathematical Cognition, 2, 95-110.

Hellige, J. B., \& Sergent, J. (1986). Role of task factors in visual field asymmetries. Brain and Cognition, 5, 200-222.

Hommel, B. (1998). Event files: Evidence for automatic integration of stimulus response episodes. Visual Cognition, 5, 183216.

Kavcic, V., \& Clarke, J. M. (2000). Hemispheric interactions during a face-word Stroop-analog task. Neuropsychology, 14, 579-587.

Koechlin, E., Naccache, L., Block, E., \& Dehaene, S. (1999). Primed numbers: Exploring the modularity of numerical representations with masked and unmasked priming. Journal of Experimental Psychology: Human Perception and Performance, 25, 1882-1905.

Lambert, A. J. (1993). Attentional interaction in the split-brain: Evidence from negative priming. Neuropsychologia, 31, 313324.

Marks, N. L., \& Hellige, J. B. (1999). Effects of bilateral stimulation and stimulus redundancy on interhemispheric interaction. Neuropsychology, 13, 475-487.

Moyer, R. S., \& Landauer, T. K. (1967). Time required for judgement of inequality. Nature, 215, 1519-1520.

Naccache, L., \& Dehaene, S. (2001). Unconscious semantic priming extends to novel unseen stimuli. Cognition, 80, 215-229.

Oldfield, R. C. (1971). The assessment and analysis of handedness: The Edinburgh inventory. Neuropsychologia, 9, 97-113.

Pesenti, M., Thioux, M., Seron, X., \& De Volder, A. (2000). Neuroanatomical substrates of Arabic number processing, numerical comparison and simple addition: A PET study. Journal of Cognitive Neuroscience, 13, 461-479.

Pinel, P., Le Clec'H, G., Van de Moortele, P. F., Naccache, L., Le Bihan, D., \& Dehaene, S. (1999). Event-related fMRI analysis of the cerebral circuit for number comparison. Neuroreport, 10 , 1473-1479.

Ratinckx, E., Brysbaert, M., \& Reynvoet, B. (2001). Bilateral field interactions and hemispheric asymmetry in number comparison. Neuropsychologia, 39, 335-345.

Reynvoet, B., \& Brysbaert, M. (1999). Single-digit and two-digit Arabic numerals address the same semantic number line. Cognition, 72, 191-201. 
Reynvoet, B., Caessens, B., \& Brysbaert, M. (in press). Automatic stimulus-response translation may be semantically mediated. Psychonomic Bulletin \& Review.

Rosselli, M., \& Ardila, A. (1989). Calculation deficits in patients with right and left hemisphere damage. Neuropsychologia, 27, 607-617.

Schwarz, W., \& Ischebeck, A. (2000). Sequential effects in number comparison. Journal of Experimental Psychology: Human Perception and Performance, 26, 1606-1621.
Sekuler, R., \& Mierkiewicz, D. (1977). Children's judgments of numerical inequality. Child Development, 48, 630-633.

Seymour, S. E., Reuter-Lorenz, P. A., \& Gazzaniga, M. S. (1994). The disconnection syndrome: Basic findings reaffirmed. Brain, 117, 105-115.

Received March 2, 2001

Revision received November 19, 2001

Accepted November 21, 2001

\section{Members of Underrepresented Groups: Reviewers for Journal Manuscripts Wanted}

If you are interested in reviewing manuscripts for APA journals, the APA Publications and Communications Board would like to invite your participation. Manuscript reviewers are vital to the publications process. As a reviewer, you would gain valuable experience in publishing. The $\mathrm{P} \& \mathrm{C}$ Board is particularly interested in encouraging members of underrepresented groups to participate more in this process.

If you are interested in reviewing manuscripts, please write to Demarie Jackson at the address below. Please note the following important points:

- To be selected as a reviewer, you must have published articles in peer-reviewed journals. The experience of publishing provides a reviewer with the basis for preparing a thorough, objective review.

- To be selected, it is critical to be a regular reader of the five to six empirical journals that are most central to the area or journal for which you would like to review. Current knowledge of recently published research provides a reviewer with the knowledge base to evaluate a new submission within the context of existing research.

- To select the appropriate reviewers for each manuscript, the editor needs detailed information. Please include with your letter your vita. In your letter, please identify which APA journal(s) you are interested in, and describe your area of expertise. Be as specific as possible. For example, "social psychology" is not sufficient-you would need to specify "social cognition" or "attitude change" as well.

- Reviewing a manuscript takes time (1-4 hours per manuscript reviewed). If you are selected to review a manuscript, be prepared to invest the necessary time to evaluate the manuscript thoroughly.

Write to Demarie Jackson, Journals Office, American Psychological Association, 750 First Street, NE, Washington, DC 20002-4242. 